\title{
Near-real-time forest fires monitoring system: case study with a manned aerial vehicle within the OSIRIS project
}

\author{
I. Reusen, N. Lewyckyj, S. Adriaensen, J. Biesemans, J. Everaerts \\ \& S. Kempenaers \\ Flemish Institute for Technological Research (VITO), \\ Remote Sensing \& Earth Observation Processes (TAP), Belgium
}

\begin{abstract}
Within the framework of the European-funded OSIRIS Integrated Project (FP6IST-2005-2.5.12 ICT for Environmental Risk Management) a high spatial resolution video sensor (with thermal capabilities) on a manned helicopter platform will be plugged-in the OSIRIS architecture to monitor forest fires in the South of France. The OSIRIS project "Open architecture for Smart and Interoperable networks in Risk Management based on In-situ Sensors" aims at enhancing the overall efficiency of the in-situ processing chain by connecting the in-situ sensors via an intelligent and versatile network infrastructure that will enable end-users to access multi-domain sensors information. During the forest fires live demonstration the video frames acquired from a helicopter platform will be down-linked in real-time to the mobile Ground Control Station (GCS) for visual inspection by the end-users and for limited processing at the $\mu$-PAF ( $\mu$ Processing and Archiving Facility). By means of a satellite link the compressed data is transferred to the Central Data Processing Center (CDPC) located at the premises of VITO in Mol (Belgium) for processing to e.g. composite images. The near-real-time, geo-referenced composite images can be used by the enduser as map of the surveyed area. The CDPC is connected to the OSIRIS architecture. Processing of data or access to archived data in the CDPC can be requested via the OSIRIS architecture. During the forest fire live demonstration planned in spring 2009 the merit of the OSIRIS concept will be evaluated against cheaper and more efficient decision-making.
\end{abstract}

Keywords: risk and disaster management, forest fires, remote sensing, nearreal-time, high spatial resolution, geo-referenced, video, infrared, UAV, PEGASUS, sensor web, open architecture. 


\section{Introduction}

Remote sensing has proved in the past to be a powerful tool for all phases in risk and disaster management (i.e. prevention/mitigation, early warning/detection, crisis/response, damage assessment). The International Charter "Space and Major Disasters" (http://www.disasterscharter.org/) [3] that enables free privileged near-real-time access to remote sensing data in case of a major disaster was activated during most of the recent major disasters worldwide (Google earth layer available at http:/www.spotimage.fr/web/en/215international-charter-on-space-and-major-disasters.php). For example the Charter was activated during the forest fires of August-September 2007 in Greece on request of the Department of Emergency Planning and Response of the Greek Civil Protection. Optical Earth Observation (EO) sensors on ENVISAT MERIS, DMC (incl. Nigeriasat-1), Landsat, SAC-C, SPOT and FORMOSAT generated a series of EO based crisis/damage mapping products for this particular crisis. Remote sensing data revealed the size (1 772655 ha or more than $12 \%$ of the total forested areas in Greece) of the disaster and plays a significant role in environmental disaster management activities on an operational basis [4]. The most recent activation of the Charter was for the cyclone Nargis in Myanmar with a provisional death toll ranging from 50000 to 100000 with 220000 people reported to be missing (Source: Reuters, 11 May 2008). The nature of the disaster and the characteristics of the object under investigation determine the optimum spatial resolution, temporal resolution and type of sensor (panchromatic, multispectral, visual, thermal, radar) to deliver the most useful remote sensing information for that particular disaster, phase or object [5].

\section{The OSIRIS project}

The OSIRIS project "Open architecture for Smart and Interoperable networks in Risk management based on In-situ Sensors" aims at enhancing the overall efficiency of the in-situ processing chain by connecting the in-situ sensors via an intelligent and versatile network infrastructure that will enable end-users to access multi-domain sensors information [1, 2]. With 13 partners and 4 endusers OSIRIS will provide architecture based on OGC (Open Geospatial Consortium, Inc. $\left.{ }^{\circledR}\right)$ standards delivering more efficient user services.

The OSIRIS architecture concept will be demonstrated in four live experiments of major environmental risks:

- Forest fires

- Industrial risks: fire in industrial buildings

- Fresh water pollution

- Air pollution in urban areas

The objective of the forest fires live demonstration is to validate the benefit of the OSIRIS architecture for the end-user. Various sensors like in-situ wireless smart imaging sensors, in-situ positioning sensors and a remote sensing system (i.e. video sensor on a manned helicopter platform) will be plugged-in the OSIRIS SWE (Sensor Web Enablement) as presented in Figure 1. 


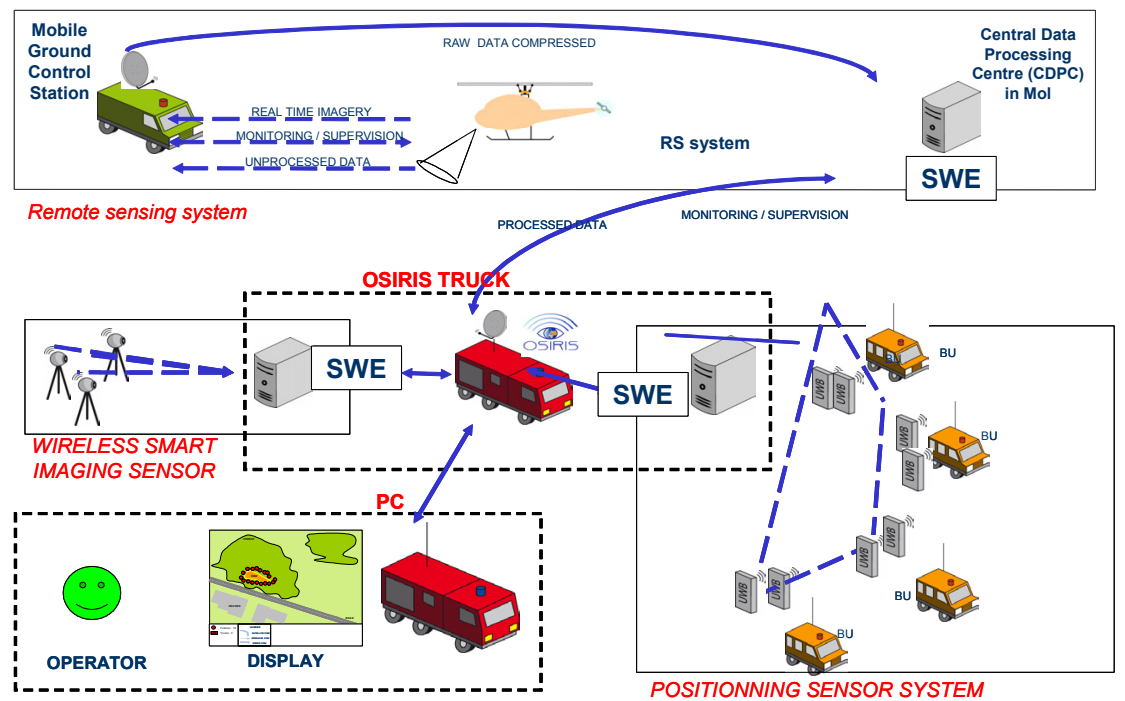

Figure 1: Forest fire live demonstration global deployment (from the OSIRIS project).

Furthermore, the OSIRIS architecture will allow that other sensors can be plugged-in easily on request of the end-user.

This paper describes the deployment of one of the sensors, a WESCAM video camera on a manned helicopter and the related data transfer and processing for the forest fire live demonstration.

The end-user requirements for the forest fire survey identified are:

Requirements: high spatial resolution $(\sim 1 \mathrm{~m})$ VIS for the survey of:

- Start of fires (ASAP)

- $\quad$ Fire front (composite image updated every 30 minutes)

- Sub-urban areas (protect the public)

- Help of rescue teams in difficulty (identify trucks, roads, ...)

- Survey of firemen control point(s)

Less priority issues:

- Large view (e.g. fire detection)

- Burned areas

- Temperature maps (fire restart)

The requirements of the end-users focus on the availability of high spatial resolution images $(\sim 1 \mathrm{~m})$ with frequent updates. An update of the fire front every 30 minutes has been requested by the French fire-fighters. Also high spatial resolution images $(\sim 30 \mathrm{~cm})$ with a high update rate (at least twice a minute) are requested for the survey of intervention teams in trouble and sub-urban areas. Images with a large view of $2-3 \mathrm{~km}$ are requested to enable to follow the dynamic of a larger fire front.

These end-user requirements were translated into requirements for the platform, the sensor and the transfer and the processing of the acquired data. 
The remote sensing system (platform/sensor/transfer/processing) selected for the forest fire demonstration is described in the next section.

\section{Remote sensing system}

This section describes the elements of the remote sensing system and how the data acquisition, data transfer and near-real-time image processing is organised during the forest fires live demonstration.

A manned helicopter of the French fire brigade equipped with a high spatial resolution digital WESCAM (MX-15) video camera with thermal capabilities, GPS and IMU (Inertial Measurement Unit) will be available for the demonstration. A mobile Ground Control Station (GCS) receives the WESCAM video data. A third party S-band system compatible with the transmitting sensor is used for data reception. A $\mu$-PAF ( $\mu$-Processing and Archiving Facility) with the following functionalities is available at the operation site to allow visual inspection of the WESCAM data and will:

- $\quad$ store all data received from the payload (images tiles, GPS data, IMU data, ...),

- decompress tiles from payload (jpeg2000 format),

- cut overlapping tiles as much as possible (normally $60 \%$ of the image),

- compose the tiles to one original image,

- compress the complete image (jpeg2000 format) reducing the amount of data,

- $\quad$ if requested, perform a composition of different images (local use - not sent),

- $\quad$ send - through satellite link - towards the CDPC.

Images are processed in the $\mu$-PAF on two dual Intel ${ }^{\circledR}$ QuadCore ${ }^{\circledR}$ systems and two fileservers providing 1TB of online storage. The images are stitched and compressed in a way that data loss is undetectable. All $\mu$-PAF electronic devices are mounted in a small, rolling 19-inch rack. Power shaping and shutdown is controlled by an uninterruptible power supply.

Tasks of the GCS and $\mu-P A F$ can be summarised as reception, buffering, processing and transmission of the data. In addition, a mobile satellite uplink system (including modulator/demodulator device, power amplifier, block up converter, low noise amplifier and $1.8 \mathrm{~m}$ quick deployable antenna) will be deployed on the operation site. This will guarantee the communication between the mobile GCS and the Central Data Processing Center (CDPC) for airborne earth observation data located at the VITO premises in Mol (Belgium). The images are then pushed towards the satellite uplink. The data is relayed over a satellite like AB3 from Eutelsat. To ensure lossless packet based transfers, standard TCP/IP solutions for all data transfers will be used. Therefore, a twoway satellite link with maximum data rate of $2 \mathrm{Mbits} / \mathrm{s}$ (uplink at the GCS and downlink at VITO) and $125 \mathrm{kbits} / \mathrm{s}$ (uplink at VITO and downlink at the GCS) will be implemented as depicted in Figure 2.

Once the compressed data has arrived at the Central Data Processing Center (CDPC), the data is archived and processed [6]. The CDPC software system 
contains an archiving workflow (Level 0 to Level 1B) and a product processing workflow for Level 1B to Level 2/3/4 (according CEOS level definition [7] with additions to the CEOS definition for level 2/3/4). The processing encompasses (a) the geo-referencing and co-registration of the imagery and (b) the production of image composites of the fire front and (c) other data requested by the end-user (e.g. continuous video stream). The CDPC hardware system consists of a cluster of 35 single and quad core dual processor machines and about 55 TB storage.

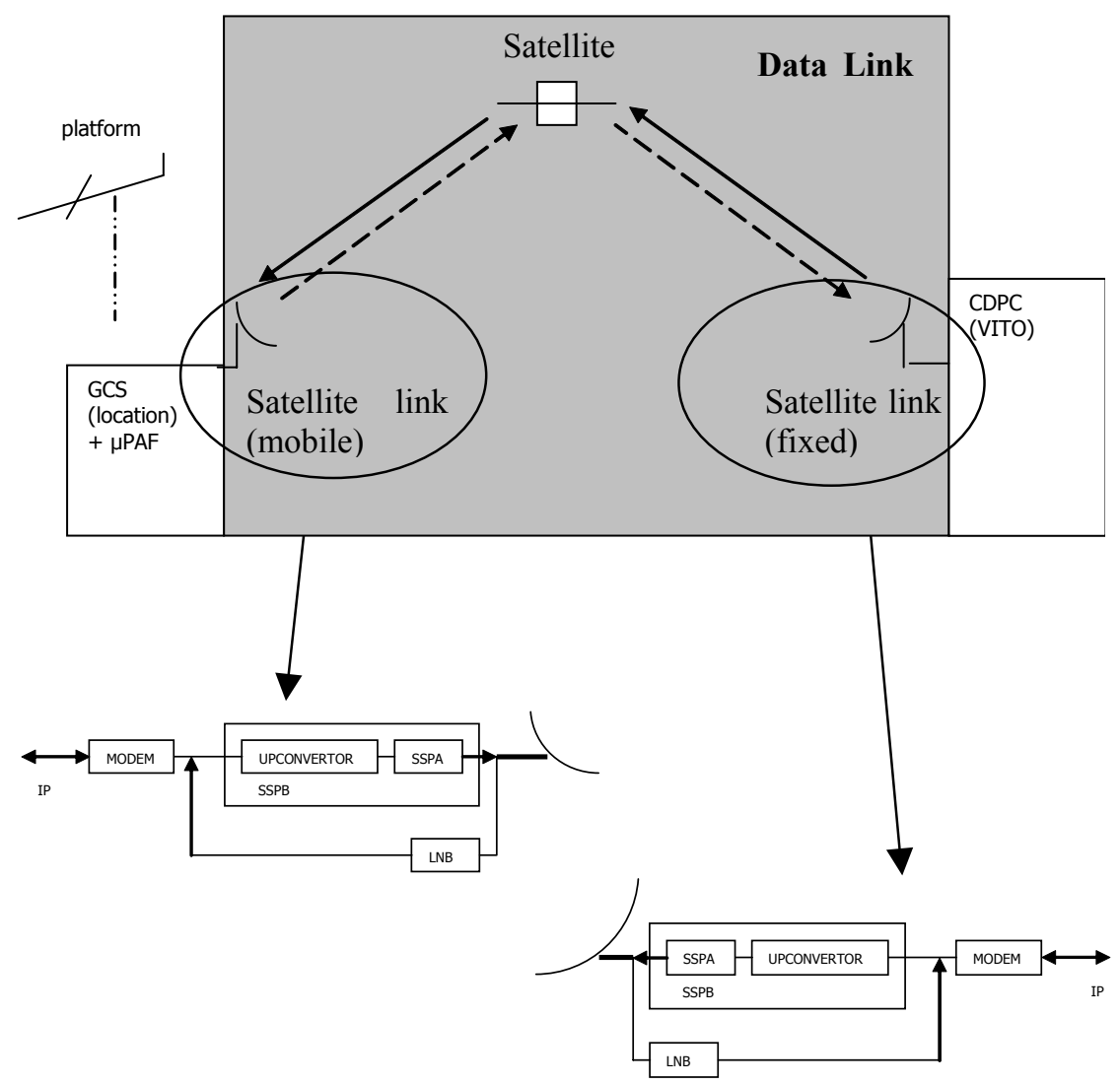

Figure 2: $\quad$ Full duplex but not capacity-symmetric satellite link (SSPA: solid state power amplifier, SSPB: block up converter, LNB: low noise block down converter).

Through OSIRIS a request for data processing or for access to archived data can be sent to the CDPC by the end-user. Near-real-time, geo-referenced and composite images will be delivered in HDF5 format (format used by NASA EOS data gateway) or JPEG2000. The produced images can be used as map by the end-user. The interface between the CDPC and OSIRIS will occur via SWE as depicted in Figure 3 compliant with OGC standards. The OGC standards that 
shall be used are WMS (Web Map Service) and WCS (Web Coverage Service) for rendering, CSW (Web Catalog Service) for discovery and SPS (Sensor Planning Service) for tasking.

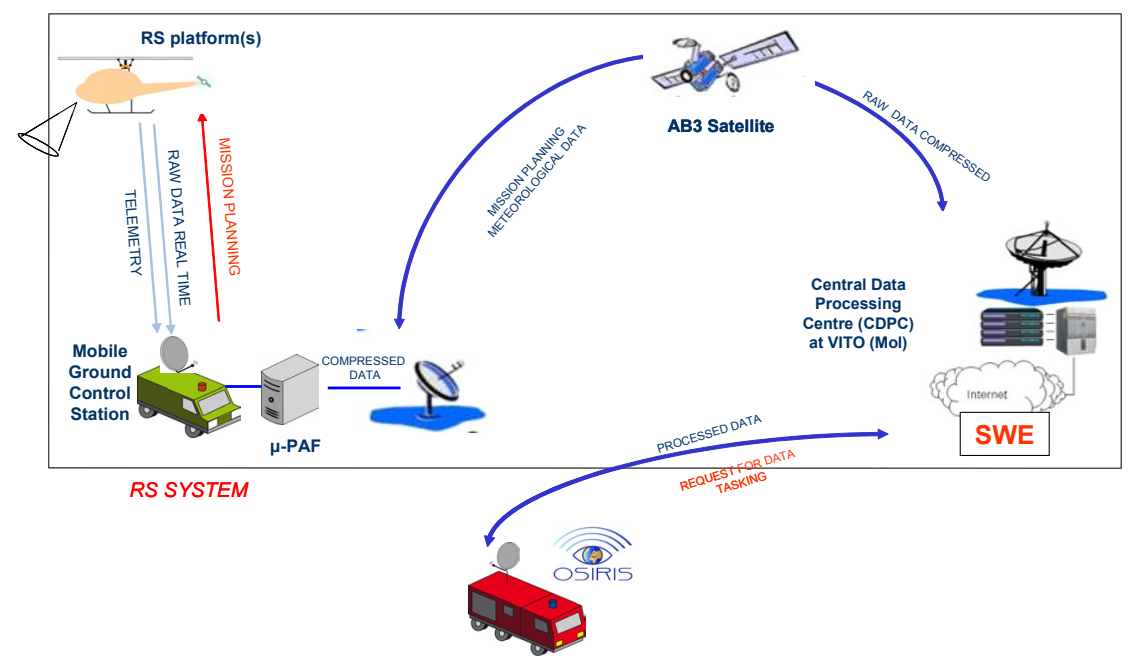

Figure 3: Description of the remote sensing system plugged-in OSIRIS.

Figure 4 shows two geo-referenced composite images of the military domain in Helchteren (Belgium) processed in near-real-time. Data was acquired on June 62006 with a QinetiQ-made video camera on a $40 \%$ scale model of the Mercator-1 Unmanned Aerial Vehicle (a demo can be downloaded from the PEGASUS website [8]).
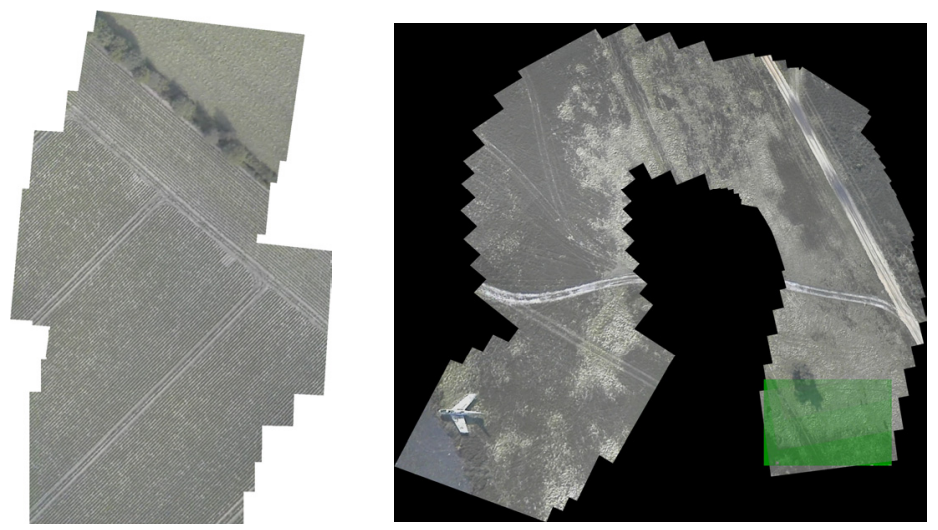

Figure 4: Geo-referenced composite images of the military domain in Helchteren (Belgium). 


\section{Live demonstration}

The forest fires live demonstration is planned for spring 2009 in the South of France in the Cévennes region.

During the live demonstration a carefully controlled forest fire with a fire line of about $3 \mathrm{~km}$ will be monitored with a manned helicopter equipped with a WESCAM (video and infrared) camera.

The described remote sensing system will be plugged-in the OSIRIS architecture to validate the capabilities of the remote sensing system integrated in the OSIRIS architecture. The remote sensing system will provide in near-realtime, high spatial resolution, geo-referenced composite images of the fire front, local high resolution images of the sub-urban regions in the vicinity of the fire, high resolution imagery of the intervention team and a global view of the infrastructure in the vicinity of the fire.

\section{Other applications}

The described remote sensing system can deliver in near-real-time high spatial resolution geo-referenced composite images. These capabilities are among the basic user requirements for many risk and disaster management applications. Therefore the described system (platform/sensor/data transfer/data processing) could be a powerful system for other risk and disaster management applications like industrial accidents, flooding, volcanic eruptions, etc.

\section{Conclusion}

The forest fires live demonstration will demonstrate that once OSIRIS and the remote sensing system is operational then a cheaper and faster solution can be offered to the envisaged forest fire end-user.

From video frames acquired from a helicopter platform, near-real-time georeferenced images are produced that can be used as map of the surveyed area at low cost.

One advantage of the OSIRIS architecture is its capability to plug-in other platforms or sensors easily.

\section{Outlook}

Once the European regulation has progressed with respect to clearance of Unmanned Aerial Vehicles (UAV), UAVs with a thermal camera on-board could be plugged-in the VITO CDPC which is connected to OSIRIS in order to better monitor forest fires. Because in risk and disaster management one often has to operate in dangerous and emergency situations, UAVs are very well suited for these situations because there is no risk for the pilot. Unmanned High Altitude/Long Endurance (HALE) platforms like the one currently being built by Verhaert [8] have an additional advantage because of their long endurance from 
weeks up to months of uninterrupted flying time. The ambitious goal of DARPA's (Defense Advanced Research Projects Agency) VULTURE program is to develop and demonstrate the ability to deliver and maintain an airborne payload on station for an uninterrupted period exceeding 5 years using a heavier than air platform system [9]. One of the concepts that will be studied is based on QinetiQ's Mercator-Zephyr platform.

\section{Acknowledgement}

OSIRIS is an Integrated Project co-funded by the INFSO DG of the European Commission within the RTD activities of the Thematic Priority Information Society Technologies.

\section{References}

[1] OSIRIS "Open architecture for Smart and Interoperable networks in Risk management based on In-situ Sensors" (Contract 0033475), Annex I

[2] OSIRIS website: http://www.osiris-fp6.eu

[3] The International Charter Space and Major Disasters website http://www.disasterscharter.org/

[4] Gitas, I. Z., Polychronaki, A., Katagis T., Mallinis, G., Contribution of remote sensing to disaster management activities: A case study of the large fires in the Peloponnese, Greece, International Journal of Remote Sensing, Vol. 29, No. 6, 20 March 2008, 1847-1853

[5] Nirupama, Simonovic, S.P., Role of Remote Sensing in Disaster Management, ICLR Research Paper Series - No. 21

[6] Biesemans J., Sterckx S., Knaeps E., Vreys K., Adriaensen S., Hooyberghs J. and Deronde B., (2007), Image processing workflows for airborne remote sensing. In: Proceedings $5^{\text {th }}$ EARSeL SIG IS Workshop, Bruges, Belgium, edited by I. Reusen

[7] CEOS website: http://www.ceos.org

[8] PEGASUS website: http://www.pegasus4europe.com/pegasus/index.htm

[9] DARPA's VULTURE program website: http://www.darpa.mil/BAA/SN0738.html 